\title{
Anticancer agent icaritin induces apoptosis through caspase-dependent pathways in human hepatocellular carcinoma cells
}

\author{
LI SUN $^{1 *}$, QISONG PENG $^{2 *}$, LILI QU ${ }^{1}$, LAILING GONG $^{1}$ and JIN SI ${ }^{1}$ \\ ${ }^{1}$ Department of Laboratory Medicine, The Second Affiliated Hospital of Nanjing Medical University, Nanjing, Jiangsu 210011; \\ ${ }^{2}$ Department of Laboratory Medicine, The Affiliated Jiangning Hospital of Nanjing Medical University, \\ Nanjing, Jiangsu 211100, P.R. China
}

Received February 20, 2014; Accepted October 31, 2014

DOI: $10.3892 / \mathrm{mmr} .2014 .3007$

\begin{abstract}
Icaritin is an active ingredient derived from the plant Herba epimedium, which exhibits various pharmacological and biological activities. However, the function, and the underlying mechanisms of icaritin on the growth of SMMC-7721 human hepatoma cells have yet to be elucidated. The present study aimed to investigate the function and underlying mechanisms of icaritin in the growth of SMMC-7721 cells. The cells were treated with varying concentrations of icaritin for 12, 24 and $48 \mathrm{~h}$, respectively, prior to cytotoxic analysis. Apoptosis of SMMC-7721 cells following treatment with icaritin was measured using flow cytometry. The gene expression of mitochondria- and Fas-mediated caspase-dependent pathways was detected by reverse transcription-quantitative polymerase chain reaction and western blotting. Statistical analysis was performed by Student's t-test and one-way analysis or variance. The present study demonstrated that treatment with icaritin significantly inhibited growth, and induced apoptosis of SMMC-7721 cells, in a time- and dose-dependent manner. In addition, icaritin triggered the mitochondrial/caspase apoptotic pathway, by decreasing the $\mathrm{Bcl}-2 / \mathrm{Bax}$ protein ratio and increasing activation of caspase-3. Icaritin also activated the Fas-mediated apoptosis pathway, as was evident by the increased expression levels of Fas and activation of caspase-8. These data suggest that icaritin may be a potent growth inhibitor and induce apoptosis of SMMC-7721 cells through the mitochondria- and Fas-mediated caspase-dependent path-
\end{abstract}

Correspondence to: Professor Jin Si, Department of Laboratory Medicine, The Second Affiliated Hospital of Nanjing Medical University, 121 Jiangjiayuan, Nanjing, Jiangsu 210011, P.R. China E-mail: sijin_efy@njmu.edu.cn

*Contributed equally

Key words: icaritin, hepatocellular carcinoma, apoptosis, mitochondrial pathway, Fas ways. The present study may provide experimental evidence for preclinical and clinical evaluations of icaritin for HCC therapy.

\section{Introduction}

Hepatocellular carcinoma (HCC) is one of the most common types of malignant tumor worldwide, and is the third leading cause of cancer-associated mortality, after lung and colon cancer (1-4). It is one of the most aggressive human malignancies and previous data have shown that the five-year survival rate is poor (5). The most effective therapy for HCC is usually surgical resection or liver transplantation; however, in $\sim 70 \%$ of patients surgery is unsuitable due to tumor metastasis or liver cirrhosis (6). The standard therapy for HCC is currently chemotherapy, particularly when surgical resection is not suitable (7). However, the current therapeutic options for HCC are not effective, since resistance and tumor relapse eventually develop (8). Novel therapeutic strategies, such as effective chemotherapy with low toxicity, are required, in order to decrease the incidence and improve the prognosis of patients with HCC.

Flavonoids are plant polyphenols that are well-known for their analgesic, physiological antipyretic and anti-inflammatory activities. Flavonoids have recently gained attention due to their antitumor activity (9-11). Icaritin is a prenylflavonoid and is a hydrolytic product of icaritin, derived from Herba Epimedii. Icaritin exhibits various pharmacological and biological activities, including antirheumatic and antidepressant effects, stimulation of cardiac and neuronal differentiation (12-13), prevention of steroid-associated osteonecrosis (14), and inhibition of growth and induction of apoptosis of PC-3 human prostate carcinoma and MCF-7 breast cancer cells (15-16).

It has recently been demonstrated that icaritin induces apoptosis of breast and endometrial cancer cells, through the mitochondrial pathway $(17,18)$. However, whether icaritin may trigger the extrinsic pathway, which is mediated by death receptors, remains unclear. The present study aimed to investigate the anticancer activities of icaritin in SMMC-7721 cells, and to identify the underlying mechanisms. 


\section{Materials and methods}

Drugs and reagents. Icaritin (purity, $>98 \%$; Fig. 1A) was purchased from Yousi Biotechnology Co., Ltd. (Shanghai, China). Dulbecco's modified Eagle's medium (DMEM), RPMI-1640 medium and newborn calf serum were purchased from Gibco Life Technologies (Carlsbad,CA, USA). An Annexin V-fluorscein isothiocyanate (FITC) Apoptosis Detection kit was purchased from Bipec Biopharma Inc. (Cambridge, MA, USA); and a Bicinchoninic Acid (BCA) Protein Quantitation kit was purchased from GENMED Scientifics Inc., USA (Shanghai, China). Primary antibodies targeting $\beta$-actin (rabbit polyclonal antibody,4967S), cleaved caspase-3 (rabbit monoclonal antibody, Asp175) and cleaved caspase-8 (mouse monoclonal antibody, Asp387) were purchased from Cell Signaling Technology, Inc. (Danvers, MA, USA). Primary antibodies targeting Bax (mouse monoclonal antibody, sc-7480) and extrinsic signal-regulated Fas (rabbit polyclonal antibody, sc-7886) were purchased from Santa Cruz Biotechnology, Inc. (Dallas, TX, USA). All of the other chemicals and solvents used in the present study were of the highest commercially available grade.

Cell culture and icaritin treatment. L02 human liver cells and SMMC-7721 human HCC cells were purchased from Nanjing KeyGen Biotech., Co., Ltd. (Nanjing, China). The cells were cultured in DMEM supplemented with $10 \%$ newborn calf serum at $37^{\circ} \mathrm{C}$ in a humidified atmosphere containing $5 \% \mathrm{CO}_{2}$.

Stock solution of icaritin, which could be further diluted with cell culture medium, was prepared in dimethyl sulfoxide (DMSO; Sigma-Aldrich, St. Louis, MO, USA) to a concentration of $10 \mathrm{mM}$, and maintained at $-20^{\circ} \mathrm{C}$. The final concentration of DMSO was $<0.1 \%$ in culture.

Cell proliferation assay. Cell proliferation was analyzed by an 3-(4, 5-dimethylthiazol-2-yl)-2, 5-diphenyltetrazolium bromide assay (Sigma-Aldrich). The L02 and SMMC-7721 cells were seeded into 96 -well plates, at a density of $5 \times 10^{3}$ cells $/ 200 \mu \mathrm{l} /$ well and incubated at $37^{\circ} \mathrm{C}$ in a humidified $5 \% \mathrm{CO}_{2}$ incubator. The cells were then treated with $0,5,10$, 20,40 or $60 \mu \mathrm{mol} / 1$ icaritin for 12,24 or $48 \mathrm{~h}$, and the plates were returned to standard tissue incubator conditions for an additional $4 \mathrm{~h}$. The media was removed and the cells were solubilized in $150 \mu \mathrm{l}$ DMSO. The color intensity of formazan was measured at a wavelength of $490 \mathrm{~nm}$, using an automated microplate spectrophotometer (iMark, Bio-Rad, Hercules, CA, USA). The survival rate of the cells was calculated using the following formula: (OD value of the treated group/OD value of untreated group) $\mathrm{x} 100 \%$. Where OD is the optical density. The assays were performed in triplicate, in three independent experiments.

Cell apoptosis assay by flow cytometry (FCM). The cells were stained with Annexin V-FITC/propidium iodide (PI) and measured using a FACSCalibur ${ }^{\mathrm{TM}}$ flow cytometer (BD FACSCanto $^{\mathrm{TM}}$ II Flow Cytometer, BD Biosciences, Franklin Lakes, NJ USA). The samples were washed twice, and adjusted to a concentration of $1 \times 10^{6}$ cells $/ \mathrm{ml}$, with phosphate-buffered saline (PBS). A total of $200 \mu \mathrm{l}$ of cell suspension was added to each tube, $5 \mu 1$ Annexin V-FITC and $10 \mu \mathrm{l}$ PI were added to the labeled tubes, and the samples were incubated for
$15 \mathrm{~min}$ at room temperature in the dark. Subsequently, $200 \mu 1$ PBS binding buffer was added to each tube and the samples were analyzed by FCM analysis (BD Biosciences) as soon as possible. The results were analyzed using FlowJo software (version 6.1.3, FlowJo, LLC, Ashland, OR, USA). The rate of apoptosis was calculated as the relative number of apoptotic cells, as compared with the total number of cells.

Reverse transcription-quantitative polymerase chain reaction $(R T-q P C R)$. The cells were frozen in liquid nitrogen and stored at $-80^{\circ} \mathrm{C}$, until further use. Bcl-2, Bax and Fas mRNA expression levels were quantified by RT-qPCR using a Reverse Transcription system (Promega Corporation, Madison, WI, USA). The reaction was conducted at $42^{\circ} \mathrm{C}$ for $60 \mathrm{~min}$.

Total cellular RNA was extracted using TRIzol ${ }^{\circledR}$ reagent (Invitrogen Life Technologies, Carlsbad, CA, USA), according to the manufacturer's instructions. The primer sequences used were as follows: Sense: 5'-CCACCAAGAAAGCAGGAAAC-3' and antisense: 5'-GCAGGATAGCAGCACAGGA-3' for Bcl-2; sense: 5'-CTGAGCTGACCTTGGAGC-3' and antisense: 5'-GACTCCAGCCACAAAGATG-3' for Bax; sense: 5'-AGCTTGGTCTAGAGTGAAAA-3' and antisense: 5'-GAGGCAGAATCATGAGATAT-3' for Fas; and sense 5'-CCTCTATGCCAACACAGTGC-3' and antisense 5'-GTACTCCTGCTTGCTGATCC-3' for $\beta$-actin. The mRNA expression levels of Bcl-2 and Bax were analyzed using one-step RT-qPCR, with RNA-direct ${ }^{\mathrm{TM}} \mathrm{SYBR}^{\circledR}$ Green Realtime PCR Master mix (Toyobo Co., Ltd., Osaka, Japan), according to the manufacturer's instructions. RNA $(2 \mu \mathrm{l})$ was amplified under the following conditions: $95^{\circ} \mathrm{C}$ for $5 \mathrm{~min}$ for denaturation followed by 40 cycles of $95^{\circ} \mathrm{C}$ for $30 \mathrm{sec}, 57^{\circ} \mathrm{C}$ for $45 \mathrm{sec}$ and $72^{\circ} \mathrm{C}$ for $1 \mathrm{~min}$. The amplification was monitored on an ABI Prism 7500 Real-Time PCR system (Applied Biosystems Life Technologies, Foster City, CA, USA).

Western blot analysis. The SMMC-7721 cells were incubated with 8,16 or $32 \mu \mathrm{M}$ icaritin for $24 \mathrm{~h}$. The cells were then lysed with ice-cold lysis buffer $(50 \mathrm{mM}$ Tris- $\mathrm{HCl}, \mathrm{pH} 7.4 ; 150 \mathrm{mM}$ $\mathrm{NaCl}, 1 \mathrm{mM} \mathrm{MgCl}{ }_{2}, 100 \mu \mathrm{g} / \mathrm{ml}$ PMSF and $1 \%$ Triton X-100) for $30 \mathrm{~min}$ on ice. The total proteins were contained within the supernatant following centrifugation at $13,225 \mathrm{x}$ g for $5 \mathrm{~min}$ at $4^{\circ} \mathrm{C}$, and the protein concentrations were measured using a BCA assay (GENMED Scientifics Inc.). Equal quantities $(40 \mu \mathrm{g})$ of lysate protein were separated on $10 \%$ SDS-PAGE gels, and electrophoretically transferred onto polyvinylidene fluoride membranes (Pall Corporation, East Hills, NY, USA). The membranes were then blocked with 5\% non-fat dry milk in TBST buffer $(10 \mathrm{mM}$ Tris, $\mathrm{pH} 7.5,150 \mathrm{mM} \mathrm{NaCl}$, and $0.05 \%$ Tween ${ }^{\circledR}-20$ ) for $2 \mathrm{~h}$ at room temperature, and probed with 1:1,000 dilutions of the primary antibodies described above, at $4^{\circ} \mathrm{C}$ overnight. Subsequently, the membranes were incubated with 1:5,000 dilutions of horseradish peroxidase-conjugated secondary antibody to mouse (Sigma-Aldrich, for the detection of Bax or cleaved caspase-8) or rabbit (Santa Cruz Biotechnology, Inc., for the detection of cleaved caspase-3, Fas and $\beta$-actin). Protein bands were visualized using an Enhanced Chemiluminescence Detection system (ChemiDoc, Bio-Rad). Band intensity was quantified using BandScan 5.0 software (Glyko, Hayward, CA, USA). All of the western blots were performed at least three times. 
A<smiles>COc1ccc(C2=C(O)C(O)c3c(O)cc(O)c(CC=C(C)C)c3O2)cc1</smiles>

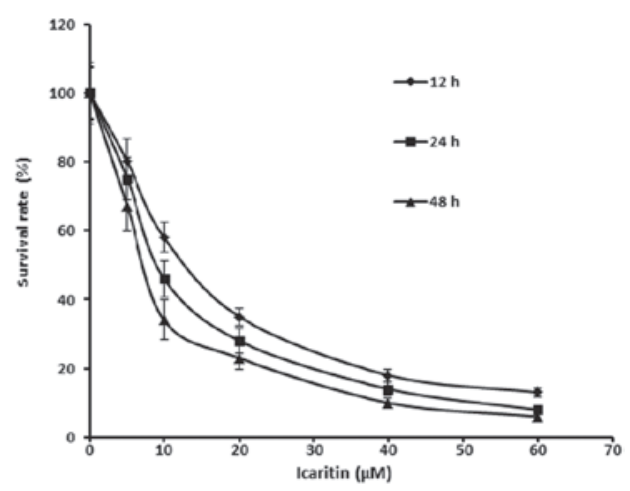

C

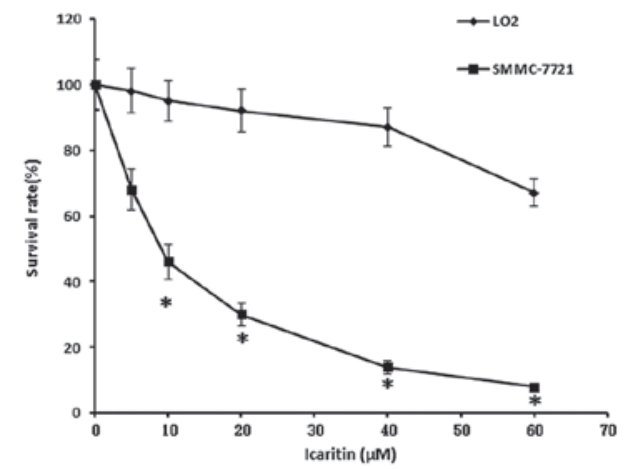

Figure 1. Icaritin exhibits growth inhibitory activity in hepatoma cells. (A) Chemical structure of icaritin. (B) Icaritin inhibited SMMC-7721 human hepatoma cells in a time- and dose-dependent manner. The cells were treated with different concentrations of icaritin for 12,24 and $48 \mathrm{~h}$, respectively, prior to cytotoxic analysis. (C) The SMMC-7721 human hepatoma cells and L02 human normal hepatocyte cells were treated with $0-60 \mu \mathrm{M}$ icaritin for $24 \mathrm{~h}$, and cytotoxicity was then assessed by a 3-(4,5-dimethylthiazol-2-yl)-2,5-diphenyltetrazolium bromide assay. Results of three independent experiments were averaged and the data are represented as the mean \pm standard error of the mean. ${ }^{*} \mathrm{P}<0.05$, compared with L02 cells.

Statistical analysis. Statistical analyses were performed using SPSS version 16.0 software (SPSS Inc., Chicago, IL, USA). Each assay was performed at least three times. Data were expressed as the mean \pm standard deviation. Student's t-test and one-way analysis of variance were used to determine statistically significant differences between the data. $\mathrm{P}<0.05$ was considered to indicate a statistically significant difference.

\section{Results}

Icaritin exhibits growth inhibitory activity on hepatoma cells, whereas the same dose causes little adverse effect on normal human liver cells. Treatment with icaritin significantly inhibited the growth of SMMC-7721 cells. The half maximal inhibitory concentration $\left(\mathrm{IC}_{50}\right)$ of icaritin in the SMMC-7721 cells, which underwent treatment for $24 \mathrm{~h}$, was $9.6 \mu \mathrm{M}$ (Table I). The rate of survival of the SMMC-7721 cells decreased in response to an increasing icaritin concentration $(0-60 \mu \mathrm{M})$, and this effect was time- and dose-dependent (Fig. 1B). Treatment with the same concentration of icaritin caused little effect on the survival of the L02 normal human liver cells (Fig. 1C). These results indicate that icaritin exerts a growth inhibitory activity on hepatoma cells, whereas the same dose did not have the same effect on normal human liver cells.

Icaritin induces significant apoptosis of SMMC-7721 cells in a time- and dose-dependent manner. Treatment with icaritin markedly induced apoptosis in the SMMC-7721 human hepatoma cells (Fig. 2A). The rate of apoptosis of the SMMC-7721
Table I. Half maximal inhibitory concentration $\left(\mathrm{IC}_{50}\right)$ of icaritin in different cell lines.

Cell line

$\mathrm{IC}_{50}$ of icaritin $(24 \mathrm{~h})$

$\mathrm{LO} 2$

$71.6 \pm 4.4$

SMMC-7721

cells was $3.0 \pm 0.3,7.8 \pm 0.9$ and $56.5 \pm 6.5 \%$, in response to treatment with 8,16 and $32 \mu \mathrm{M}$ icaritin, respectively. However, 0-32 $\mu \mathrm{M}$ icaritin did not trigger significant levels of apoptosis in the L02 human normal hepatocyte cells (Fig. 2B and C). In addition, icaritin induced an increased rate of apoptosis in a dose-dependent manner, when the time of treatment was fixed (Fig. 2D). These results suggest that treatment with icaritin markedly induced apoptosis of SMMC-7721 HCC cells in a time- and dose-dependent manner.

Icaritin upregulates the protein expression levels of Bax and cleaved caspase-3 and effects gene expression in SMMC-7721 cells. Treatment of the SMMC-7721 cells with various concentrations of icaritin for $24 \mathrm{~h}$ significantly increased the protein expression levels of Bax and cleaved caspase-3 (Fig. 3A). The protein expression levels of Bax and cleaved caspase- 3 , at the mitochondrial membrane, were significantly increased in response to treatment with icaritin, in a dose-dependent manner (Fig. 3B). Furthermore, 
A

SMMC-7721
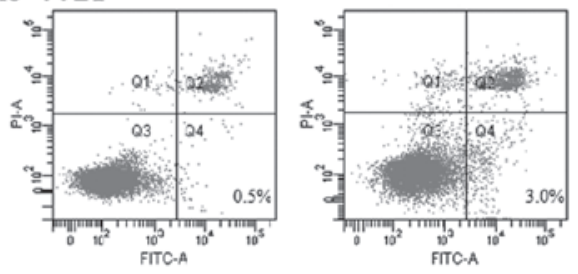

$0 \mu \mathrm{mol} / 1$

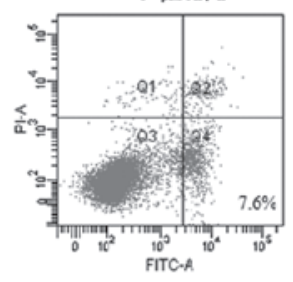

$16 \mu \mathrm{mol} / 1$

$\mathbf{C}$

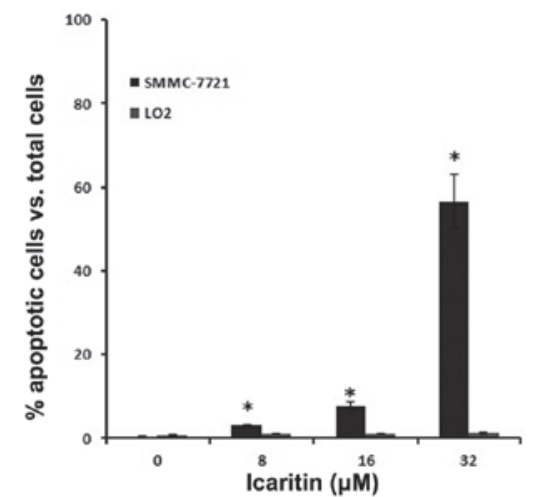

B
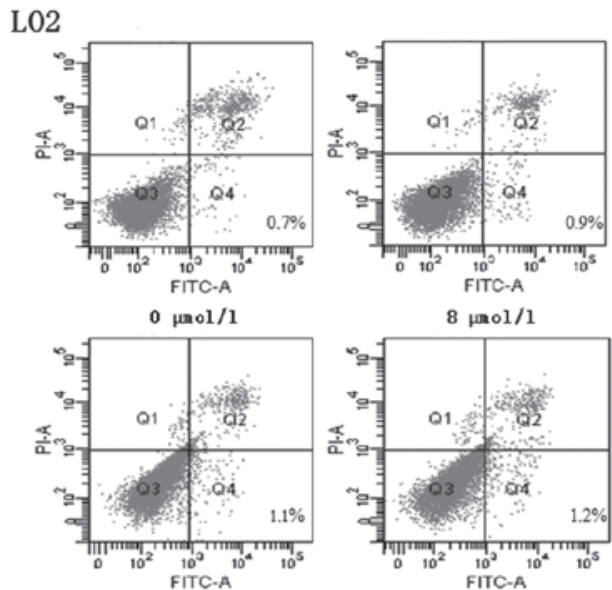

$16 \mu \mathrm{nol} / 1$

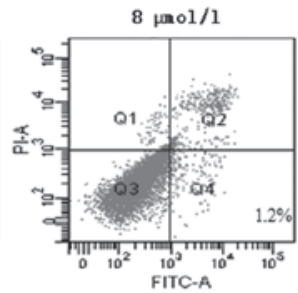

$32 \mu=01 / 1$

D

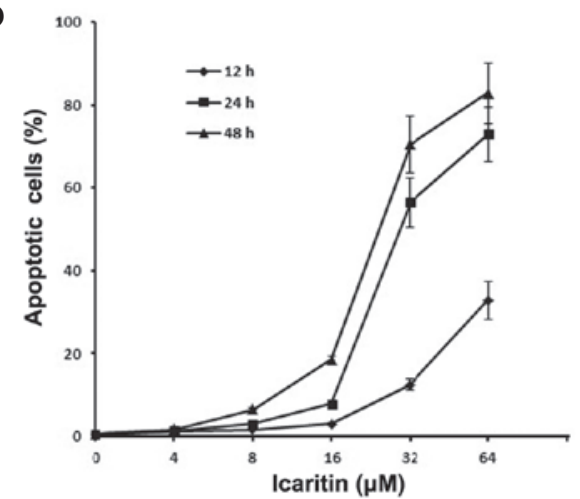

Figure 2. Icaritin induces significant apoptosis of SMMC-7721 human hepatoma cells. (A) Apoptosis of SMMC-7721 cells was assessed $24 \mathrm{~h}$ after treatment with 8-32 $\mu \mathrm{M}$ icaritin, by Annexin V-fluorescein isothiocyanate/propidium iodide binding and measured by flow cytometry (FCM). The rate of apoptosis was analyzed. (B) L02 normal hepatocyte cells were treated with under same conditions as SMMC-7721 cells and then assayed by FCM. (C) Icaritin induces significant apoptosis of SMMC-7721 cells. "P $<0.05$, compared with L02 cells. (D) There was a positive correlation between the percentage of apoptotic SMMC-7721 cells and the dose of icaritin, or treatment time. Results of three independent experiments were averaged and the data are represented as the mean \pm standard error of the mean.

A

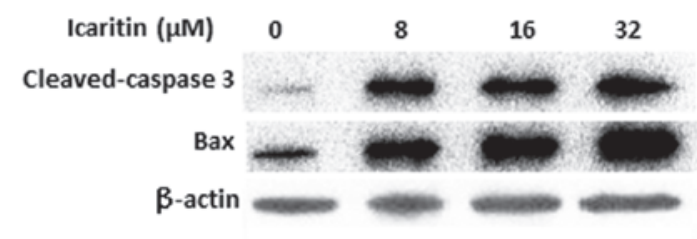

C
B

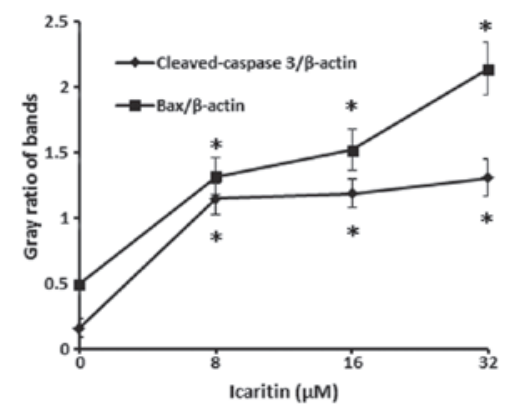

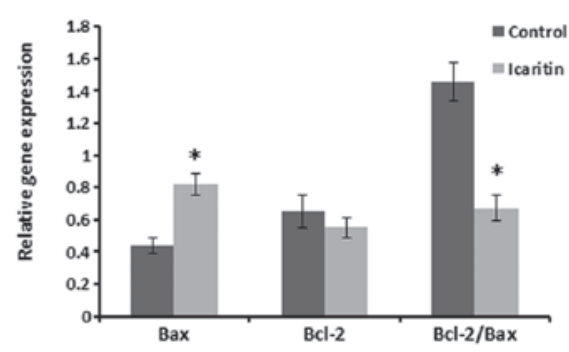

Figure 3. Effects of icaritin on the expression levels of apoptotic genes in SMMC-7721 human hepatoma cells. (A) Bax and cleaved caspase-3 protein expression was determined in SMMC-7721 cells by western blot analysis $24 \mathrm{~h}$ after treatment with 8-32 $\mu \mathrm{M}$ icaritin. (B) Gray analysis of protein bands. "P<0.05, compared with control untreated group. (C) Bcl-2 family gene expression levels were analyzed by reverse transcription-quantitative polymerase chain reaction. mRNA expression levels of Bax and Bcl-2 were quantified relative to $\beta$-actin mRNA expression. Treatment with icaritin decreased Bcl-2 and increased Bax mRNA expression levels, resulting in a decreased Bcl-2/Bax ratio. " $\mathrm{P}<0.05$, compared with control untreated group. Results of three independent experiments were averaged and the data are represented as the mean \pm standard error of the mean. 
A

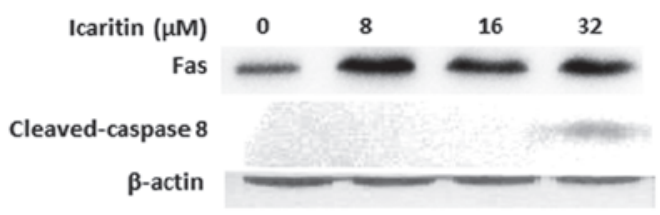

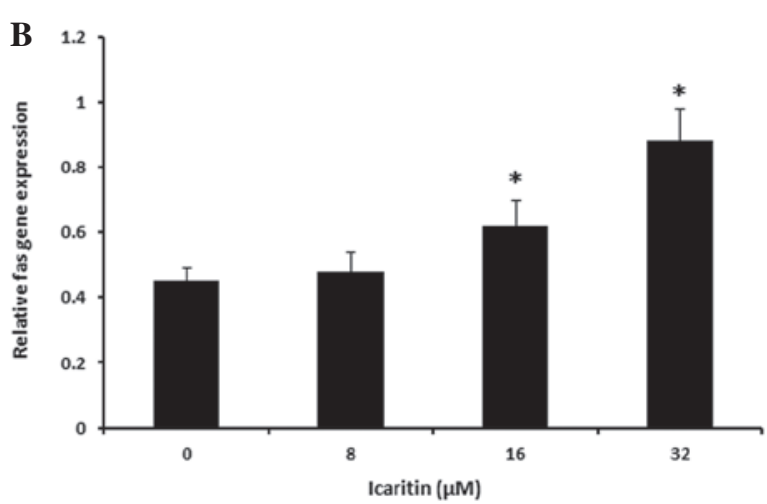

Figure 4. Icaritin triggered the extrinsic Fas-mediated apoptotic pathway in SMMC-7721 human hepatoma cells. (A) Protein expression levels of Fas and cleaved caspase-8 in SMMC-7721 cells were determined by western blot analysis $24 \mathrm{~h}$ after treatment with $8-32 \mu \mathrm{M}$ icaritin. The expression levels of Fas were increased following treatment with icaritin. However, treatment of SMMC-7721 cells with icaritin for 24 h did not induce cleaved caspase- 8 protein expression, until the concentration of icaritin had reached $32 \mu \mathrm{M}$. (B) Fas gene expression levels were analyzed by reverse transcription-quantitative polymerase chain reaction. The mRNA expression levels of Fas were quantified relative to $\beta$-actin mRNA expression. Treatment with icaritin resulted in increased mRNA expression levels of Fas. ${ }^{*} \mathrm{P}<0.05$, compared with the control untreated group. Results of three independent experiments were averaged and the data are represented as the mean \pm standard error of the mean.

treatment with icaritin decreased Bcl-2 and increased Bax mRNA expression levels, resulting in a decreased Bcl-2/Bax ratio (Fig. 3C).

Effects of icaritin on SMMC-7721 apoptosis, by the extrinsic pathway. The results of the present study indicate that icaritin may trigger the mitochondrial pathway of apoptosis in SMMC-7721 cells (Fig. 3). The present study also aimed to determine whether icaritin could induce apoptosis through the death receptor pathway. The protein expression levels of Fas were increased following treatment with icaritin (Fig. 4A). Treatment of the SMMC-7721 cells with $32 \mu \mathrm{M}$ icaritin for $24 \mathrm{~h}$ also induced cleaved caspase- 8 protein expression. Icaritin also upregulated the mRNA expression levels of Fas in the SMMC-7721 cells (Fig. 4B).

\section{Discussion}

HCC is the third most common cause of cancer-associated mortality, with $\sim 600,000$ fatalities worldwide (19). The most effective therapy for HCC is surgical resection; however, $<15 \%$ of patients can benefit from this treatment, due to the presence of numerous tumor nodules. Until recently, chemotherapy for patients with HCC was toxic and not particularly effective. Thus emphasizing the requirement for further research into the molecular mechanisms of HCC development, and identification of non-cytotoxic and effective antineoplastic compounds for chemoprevention and treatment. Recently, more traditional herbal medicines are being considered for use as anticancer treatments (20-22). However, few anticancer agents with low toxicity have been identified to be safe and effective for the treatment of HCC. It has previously been demonstrated that certain Chinese natural ingredients, or herbal formulas, have preventive and therapeutic effects against cancer. In particular, the development of icaritin has gained attention as an antifibrotic and antineoplastic monomer, purified from the herb Epimedium (23-25). The present study demonstrated that icaritin may inhibit growth and significantly induce apoptosis in SMMC-7721 cells, with an effective concentration range between 8 and $32 \mu \mathrm{M}$. The icaritin-induced growth inhibition and apoptosis were time- and dose-dependent. Furthermore, icaritin at the concentrations mentioned above, hardly affected the growth and apoptosis of L02 non-tumor human hepatocyte cells. These results suggest that icaritin may possess a selective antitumor action.

The present study also investigated the underlying mechanisms of icaritin-induced apoptosis of HCC cells. The expression of a number of genes, including Bcl-2, Bax and Caspase- 3 have been revealed to be altered following treatment with icaritin, but the study, which demonstrated this was focussed on the mitochondrial pathway (26). Bax and Bcl-2 family members are critical regulators of mitochondrial function (27-30). They may activate or inhibit the release of cytochrome $c$, which leads to the activation of caspase- 3 in the process of apoptosis. Bax exerts pro-apoptotic activity by translocation from the cytosol to the mitochondria, and induces the release of cytochrome $c$; whereas Bcl-2 exerts anti-apoptotic activity by inhibiting the translocation of Bax to the mitochondria (31). The $\mathrm{Bcl}-2$ / Bax ratio is correlated with the extent of apoptosis, and is a crucial factor in the induction of apoptosis (32). In the present study, treatment with icaritin resulted in increased Bax and decreased Bcl-2 expression levels, leading to a decreased Bcl-2/Bax ratio. Caspase-3 protein was cleaved and cleaved caspase- 3 expression levels were shown to be increased. The alterations to the Bcl-2/Bax ratio and the expression of cleaved caspase-3, in response to treatment with icaritin, were dose-dependent.

Mitochondria have a pivotal role in the signal transduction of apoptosis (33); however, activation of the mitochondrial pathway is not the only mechanism by which icaritin may induce the apoptosis of HCC cells. The extrinsic pathway is mediated by death receptors, and involves Fas and the binding and activation of caspase-8 (34-36). It has previously been reported that the engagement of Fas may promote the formation of the death-inducing signaling complex, resulting in activated T cell apoptosis (37). The Fas ligand activates caspase-8, following interaction with the Fas receptor (38). Activation of 
caspase- 8 may lead to the activation of downstream effector proteases, such as caspase-3, resulting in apoptosis (39-40). The present study showed that the expression levels of Fas were significantly increased following treatment with icaritin and the protein expression levels of cleaved caspase- 8 were detected following treatment with $32 \mu \mathrm{M}$ icaritin. These results demonstrate that increased apoptosis may be achieved through the extrinsic pathway in SMMC-7721 cells treated with icaritin.

The results of the present study indicate that icaritin may inhibit growth and induce apoptosis in SMMC-7721 cells. These effects were dose-dependent and not observed in L02 normal hepatocyte cells. The underlying mechanisms of icaritin-induced apoptosis were also examined. The results of the present study indicate that icaritin-induced apoptosis involves the intrinsic mitochondria and extrinsic death receptor pathways. Fas activation was shown to be required for icaritin-induced apoptosis. These data suggest that icaritin may be developed as a promising anticancer agent against human HCC.

\section{Acknowledgements}

This study was supported by the National Natural Science Foundation of China (grant no. 81472831), the Medical Key Talent Foundation of Jiangsu Province (grant no. RC2011081), the Medical Key Science and Technology Development Projects of Nanjing (grant no. ZKX11176) and the Science and Technology Development Foundation of Nanjing Medical University of China (grant no. 2011NJMU150).

\section{References}

1. McKillop IH, Moran DM, Jin X and Koniaris LG: Molecular pathogenesis of hepatocellular carcinoma. J Surg Res 136 125-135, 2006

2. Bruix J, Boix L, Sala M and Llovet JM: Focus on hepatocellular carcinoma. Cancer Cell 5: 215-219, 2004.

3. Trevisani F, Cantarini MC, Wands JR and Bernardi M: Recent advances in the natural history of hepatocellular carcinoma. Carcinogenesis 29: 1299-1305, 2008.

4. Kusakabe A, Tanaka Y, Orito E, et al: A weak association between occult HBV infection and non-B non-c hepatocellular carcinoma in Japan. J Gastroenterol 42: 298-305, 2007.

5. El-Serag HB, Siegel AB, Davila JA, Shaib YH, Cayton-Woody M, McBride R and McGlynn KA: Treatment and outcomes of treating of hepatocellular carcinoma among Medicare recipients in the United States: a population-based study. J Hepatol 44: 158-166, 2006.

6. Llovet JM, Burroughs A and Bruix J: Hepatocellular carcinoma. Lancet 362: 1907-1917, 2003.

7. Plataniotis G and Castiglione M; ESMO Guidelines Working Group: Endometrial cancer : ESMO Clinical Practice Guidelines for diagnosis, treatment and follow-up. Ann Oncol 2: v41-v45, 2010.

8. Gehrig PA and Bae-Jump VL: Promising novel therapies for the treatment of endometrial cancer. Gynecol Oncol 116: 187-194, 2010.

9. Middleton E Jr, Kandaswami C and Theoharides TC: The effects of plant flavonoids on mammalian cells: implications for inflammation, heart disease, and cancer. Pharmacol Rev 52: 673-751, 2000.

10. Li YL, Gan GP, Zhang HZ, Wu HZ, Li CL, Huang YP, Liu YW and Liu JW: A flavonoid glycoside isolated from Smilax china L. rhizome in vitro anticancer effects on human cancer cell lines J Ethnopharmacol 113: 115-124, 2007.

11. Díaz JG, Carmona AJ, Torres F, Quintana J, Estévez F and Herz W: Cytotoxic activities of flavonoid glycoside acetates from Consolida oliveriana. Planta Med 74: 171-174, 2008.
12. Wang Z, Wang $\mathrm{H}$, Wu J, Zhu D, Zhang $\mathrm{X}$, Ou L, Yu Y and Lou Y: Enhanced co-expression of beta-tubulin III and choline acetyltransferase in neurons from mouse embryonic stem cells promoted by icaritin in an estrogen receptor-independent manner. Chem Biol Interact 179: 375-385, 2009.

13. Wo YB, Zhu DY, Hu Y, Wang ZQ, Liu J and Lou YJ: Reactive oxygen species involved in prenylflavonoids, icariin and icaritin, initiating cardiac differentiation of mouse embryonic stem cells. J Cell Biochem 103: 1536-1550, 2008.

14. Zhang G, Qin L, Sheng H, Wang XL, Wang YX, Yeung DK, Griffith JF, Yao XS, Xie XH, Li ZR, Lee KM and Leung KS: A novel semisynthesized small molecule icaritin reduces incidence of steroid-associated osteonecrosis with inhibition of both thrombosis and lipid-deposition in a dose-dependent manner. Bone 44: 345-356, 2009.

15. Huang X, Zhu D and Lou Y: A novel anticancer agent, icaritin, induced cell growth inhibition, Gl arrest and mitochondrial transmenbrane potential drop in human prostate carcinoma PC-3 cells. Eur J Pharmacol 564: 26-36, 2007.

16. Wang ZQ and Lou YJ: Proliferation-stimulating effects of icaritin and desmethylicaritin in MCF-7 cells. Eur J Pharmacol 504: 147-153, 2004.

17. Guo Y, Zhang X, Meng J and Wang ZY: An anticancer agent icaritin induces sustained activation of the extracellular signal-regulated kinase (ERK) pathway and inhibits growth of breast cancer cells. Eur J Pharmacol 658: 114-122, 2011.

18. Tong JS, Zhang QH, Huang X, Fu XQ, Qi ST, Wang YP, Hou Y, Sheng J and Sun QY: Icaritin causes sustained ERK1/2 activation and induces apoptosis in human endometrial cancer cells. PLoS One 6: e16781, 2011

19. Parkin DM, Bray F, Ferlay J and Pisani P: Global cancer statistics, 2002. CA Cancer J Clin 55: 74-108, 2005.

20. Zhou NN, Tang J, Chen WD, Feng GK, Xie BF, Liu ZC, Yang D and Zhu XF: Houttuyninum, an active constituent of Chinese herbal medicine, inhibits phosphorylation of HER2/neu receptor tyrosine kinase and the tumor growth of HER2/neu-overexpressing cancer cells. Life Sci 90: 770-775, 2012.

21. Wang CZ, Calway T and Yuan CS: Herbal medicines as adjuvants for cancer therapeutics. Am J Chin Med 40: 657-669, 2012.

22. Ben-Arye E, Schiff E, Hassan E, Mutafoglu K, Lev-Ari S, Steiner M, Lavie O, Polliack A, Silbermann M and Lev E: Integrative oncology in the Middle East: from traditional herbal knowledge to contemporary cancer care. Ann Oncol 23: 211-221, 2012.

23. Li J, Liu P, Zhang R, Cao L, Qian H, Liao J, Xu W, Wu M and Yin Z: Icaritin induces cell death in activated hepatic stellate cells through mitochondrial activated apoptosis and ameliorates the development of liver fibrosis in rats. J Ethnopharmacol 137: 714-723, 2011.

24. Zhou J, Wu J, Chen X, Fortenbery N, Eksioglu E, Kodumudi KN, Pk EB, Dong J, Djeu JY and Wei S: Icariin and its derivative, ICT, exert anti-inflammatory, anti-tumor effects, and modulate myeloid derived suppressive cells (MDSCs) functions. Int Immunopharmacol 11: 890-898, 2011.

25. Huang J, Yuan L, Wang X, Zhang TL and Wang K: Icaritin and its glycosides enhance osteoblastic, but suppress osteoclastic, differentiation and activity in vitro. Life Sci 81: 832-840, 2007.

26. He J, Wang Y, Duan F, Jiang H, Chen MF and Tang SY: Icaritin induces apoptosis of HepG2 cells via the JNK1 signaling pathway independent of the estrogen receptor. Planta Med 76: 1834-1839, 2010.

27. Golbano JM, Lóppez-Aparicio P, Recio MN and Pérez-Albarsanz MA: Finasteride induces apoptosis via Bcl-2, Bcl-xL, Bax and caspase-3 proteins in LNCaP human prostate cancer cell line. Int J Oncol 32: 919-924, 2008.

28. Kroemer G and Reed JC: Mitochondrial control of cell death. Nat Med 6: 513-519, 2000.

29. van Loo G, Saelens X, van Gurp M, MacFarlane M, Martin SJ and Vandenabeele P: The role of mitochondrial factors in apoptosis: a Russian roulette with more than one bullet. Cell Death Differ 9: 1031-1042, 2002.

30. Llambi F, Moldoveanu T, Tait SW, Bouchier-Hayes L, Temirov J, McCormick LL, Dillon CP and Green DR: A unified model of mammalian BCL-2 protein family interactions at the mitochondria. Mol Cell 44: 517-531, 2011.

31. Wang X: The expanding role of mitochondria in apoptosis. Genes Dev 15: 2922-2933, 2001.

32. Susnow N, Zeng L, Margineantu D and Hockenbery DM: Bcl-2 family proteins as regulators of oxidative stress. Semin Cancer Biol 19: 42-49, 2009. 
33. Tait SW and Green DR: Mitochondria and cell death: outer membrane permeabilization and beyond. Nat Rev Mol Cell Biol 11: 621-632, 2010.

34. Fulda S and Debatin KM: Extrinsic versus intrinsic apoptosis pathways in anticancer chemotherapy. Oncogene 25: 4798-4811, 2006.

35. Yamaguchi Y, Shiraki K, Fuke H, Inoue T, Miyashita K, Yamanaka Y and Nakano T: Adenovirus-mediated transfection of caspase-8 sensitizes hepatocellular carcinoma to TRAIL- and chemotherapeutic agent-induced cell death. Biochim Biophys Acta 1763: 844-853, 2006.

36. Wang SH, Chen LM, Yang WK and Lee JD: Increased extrinsic apoptotic pathway activity in patients with hepatocellular carcinoma following transarterial embolization. World $\mathrm{J}$ Gastroenterol 17: 4675-4681, 2011.

37. Muppidi JR and Siegel RM: Ligand-independent redistribution of Fas (CD95) into lipid rafts mediates clonotypic T cell death. Nat Immunol 5: 182-189, 2004
38. Abd El-Ghany RM, Sharaf NM, Kassem LA, Mahran LG and Heikal OA: Thymoquinone triggers anti-apoptotic signaling targeting death ligand and apoptotic regulators in a model of hepatic ischemia reperfusion injury. Drug Discov Ther 3: 296-306, 2009.

39. Hyer ML, Shi R, Krajewska M, Meyer C, Lebedeva IV, Fisher PB and Reed JC: Apoptotic activity and mechanism of 2-cyano-3,12-dioxoolean-1,9-dien-28-oic-acid and related synthetic triterpenoids in prostate cancer. Cancer Res 68: 2927-2933, 2008.

40. Zhang L, Zhang Y, Zhang L, Yang X and Lv Z: Lupeol, a dietary triterpene, inhibited growth, and induced apoptosis through down-regulation of DR3 in SMMC7721 cells. Cancer Invest 27: 163-170, 2009. 